\title{
GEOMETRÍA FLEXIBLE PARA LAS ESTRUCTURAS DE BARRAS
}

\author{
(FLEXIBLE GEOMETRY FOR BAR STRUCTURES)
}

Luis Sánchez-Cuenca López, Dr. arquitecto

Universitat de Girona. Escola Politècnica Superior

Departamento de Arquitectura e Ingeniería de la Construcción

ESPAÑA
Fecha de recepción: $15-$ II -94

\begin{abstract}
RESUMEN
En este articulo se hace una propuesta geométrica que puede ser aplicable a las estructuras de barras. Se parte de sustituir en una trama estructural sus nudos habituales por un sistema de empalmes de barras dos a dos, lo que se consigue girando en cada mudo las barras que en él concurren. La aparición de esfuerzos de flexión y la introducción de articulaciones en cada uno de estos empalmes permiten pasar de una geometría rígida o indeformable a una "flexible", de manera que una misma trama estructural pueda adoptar diferentes tamaños conservando su forma geométrica original.
\end{abstract}

En el artículo se analizan los parámetros geométricos que ban de ponerse en juego para que el proceso resulte coherente.

\section{SUMMARY}

This article brings a geometric proposal which can be applied to the bar structures. The starting point is the substitution of the usual knots in a structural web by a system of combining the bars two by two, which is achieved by twisting the bars in each knot. The tensile forces that appear and the introduction of joints in each of these knots allow the transition from a rigid or undeformable geometry to a new "flexible" one leading to the possibility of one and the same structural web adopting different sizes while preserving its original geometric form.

The article analyzes the geometric parametres to be used in order to obtain a coherent process.

\section{Nudo y empalme}

Las estructuras de barras, ya sean de una o de varias capas, se organizan, por lo general, según una geometría rígida o indeformable mediante diversos tipos de triangulaciones, lo que implica nudos en los que concurren desde tres barras, como mínimo, hasta cinco, seis u ocho barras.

Una alternativa a este nudo (de $n$ barras) es su transformación en un sistema de $n$ empalmes de barras dos a dos.
La Fig. 1 muestra cómo un nudo (en este caso de cuatro barras) puede transformarse en un sistema de cuatro empalmes de barras dos a dos, de forma que el vértice del nudo se convierte en un polígono de tantos lados como barras concurrían en el nudo. Esta transformación se consigue mediante la aplicación de un cierto giro a cada una de las barras del nudo, y en ese caso el polígono que se genera será tanto mayor cuanto mayor sea el giro aplicado a las barras.

Lo que caracteriza al empalme y lo diferencia del nudo es que así como en el nudo los extremos de 

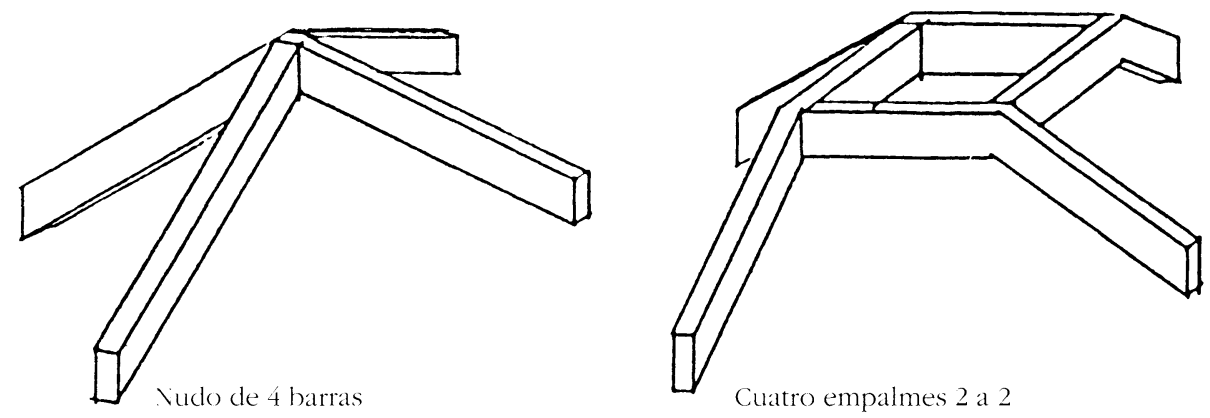

Fig. 1

las barras concurren en el vértice, en el empalme, en cambio, la unión (siempre entre sólo dos barras) se realiza entre el extremo de una y un punto intermedio de la otra, de manera que en esta última barra se producen esfuerzos de flexión, cuando con los nudos habituales no se producen en las barras más que esfuerzos de compresión o tracción.

Estos esfuerzos de flexión parecen en principio un grave inconveniente. Sin embargo, si en cada barra se introduce una articulación en el punto del empalme, los esfuerzos de flexión se transformarán en giros, con lo que toda la trama estructural pierde parte de su rigidez y entra en una situación de "geometría variable", que aquí se ha denominado "flexible" puesto que, como se verá, puede variar sólo entre unos límites muy concretos.

La propuesta tiene relación, pues, con la geometría variable y tiene algunos puntos de contacto con la "tensegrity" de Buckminster Fuller.

\section{Modelo geométrico}

Para que el proceso sea coherente es necesario que se cumplan algunas condiciones:

1. La trama ha de poder asimilarse a una superficie directriz envolvente, cuya forma (aunque no su tamaño) se va a conservar durante todo el proceso.

2. El eje de giro en cada empalme debe ser perpendicular en ese punto a la superficie directriz inicial. Las variaciones que exigirá el desarrollo del proceso pueden trasladarse después a las propias barras.

3. Los giros de las barras en nudos similares han de ser idénticos en magnitud y sentido.
El control de la segunda condición resulta muy sencillo si la superficie directriz es esférica. En ese caso los ejes de giro de toda la trama pasarán necesariamente por el centro de esa esfera envolvente inicial. A lo largo del proceso, y al modificarse el radio de la superficie esférica envolvente, las variaciones exigibles al eje de giro pueden trasladarse a la propia barra.

Por ello, el modelo geométrico elegido como punto de partida ha sido el conjunto de los poliedros regulares, todos ellos inscribibles en una esfera. Una generalización posterior permitirá considerar el conjunto de los poliedros arquimedianos y sus conjugados, así como los desarrollos geodésicos que de unos y otros puedan derivarse. Otra generalización más amplia permitirá contemplar tramas estructurales no necesariamente esféricas.

En lo que sigue dejaremos de hablar de tramas, barras y nudos, que sustituiremos por poliedros, aristas y vértices.

\section{Descripción del proceso}

Como procedimiento para sustituir los vértices por empalmes se ha utilizado el giro de las aristas. Las aristas giran sobre su punto medio alrededor de un eje que pasa por él y por el centro del poliedro y de su esfera circunscrita. Fig. 2.1.

Una vez giradas las aristas quedarían desconectadas. Para asegurar su conexión se utilizan los pasos siguientes:

1. Se elige un poliedro base (en este caso se ha partido del icosaedro regular).

2. Las aristas se convierten en arcos geodésicos de su esfera circunscrita (Fig. 2.2), arcos cuyas cuerdas serán las aristas. 

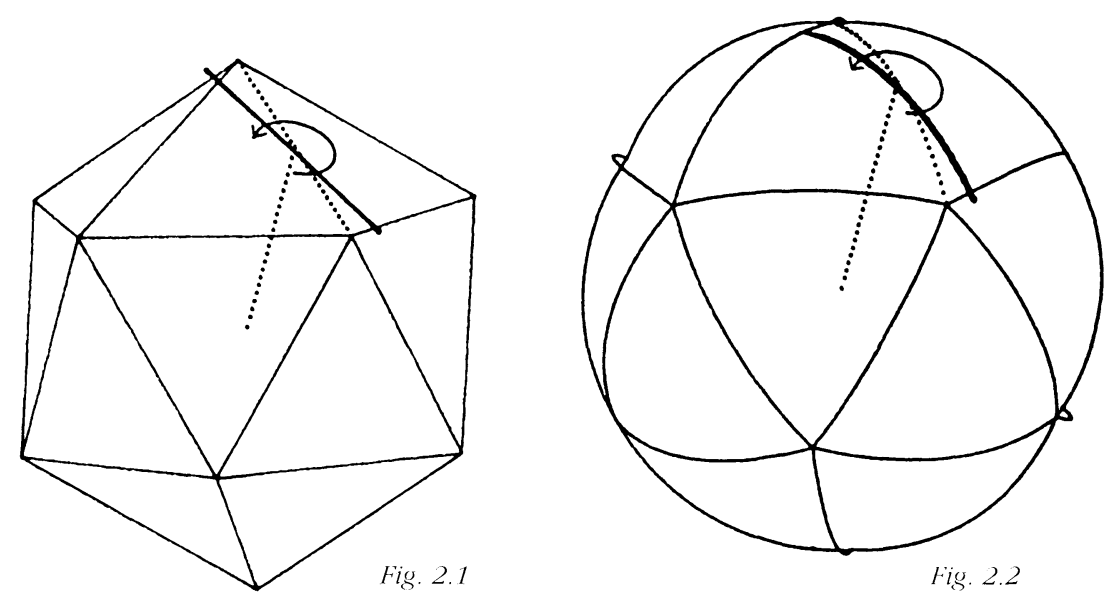

Fig. 2.2
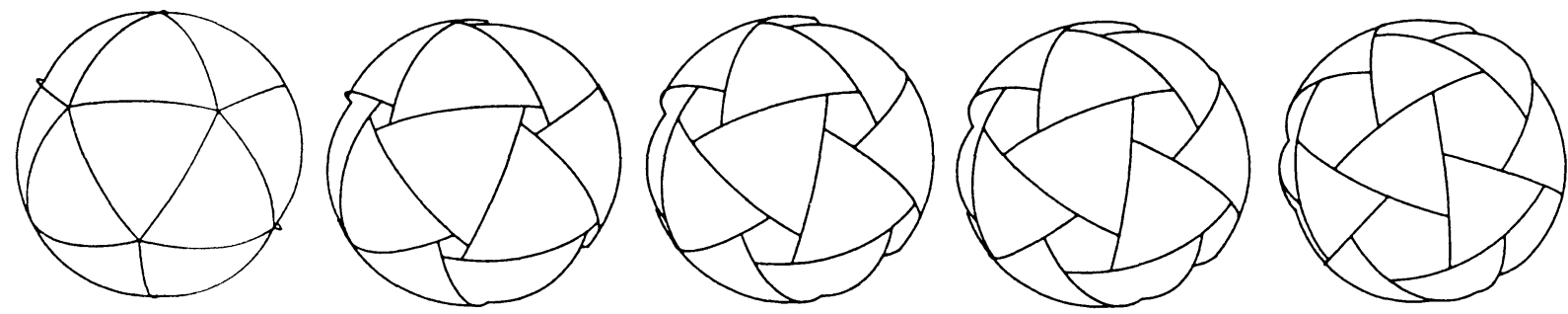

Iig. 2.;
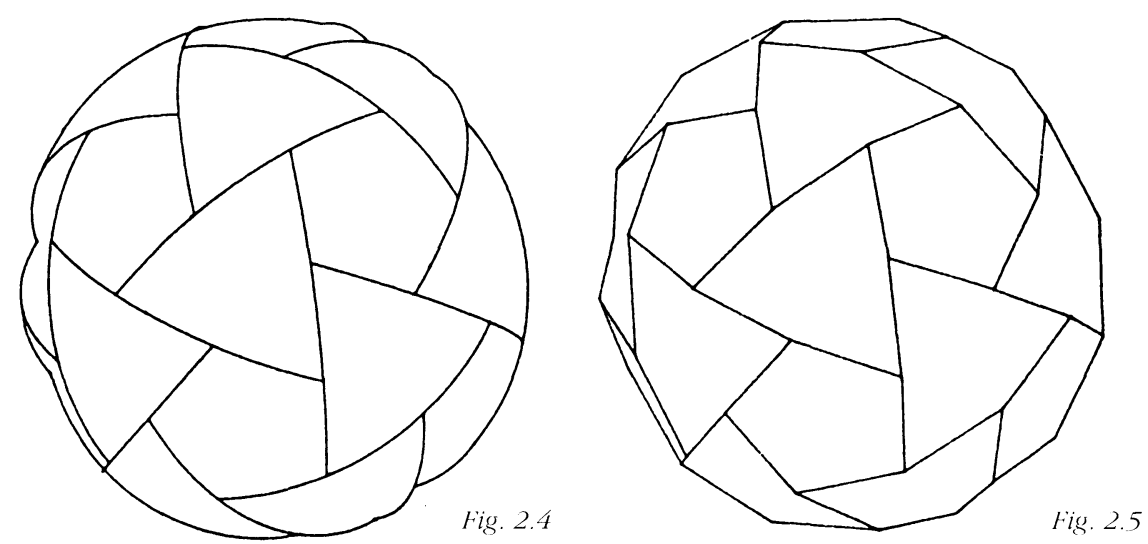

3. Se giran estos arcos alrededor del radio que pasa por su centro, y se prolongan de forma que se asegure su intersección. Esta intersección siempre será posible puesto que los arcos, una vez girados, siguen estando sobre la misma superficie esférica.

4. Cada vértice se descompondrá en un polígono de tantos lados como arcos concurrían en él. Este polígono será tanto mayor cuanto mayor sea el ángulo de giro de los arcos. Fig. 2.3.

5. Al mismo tiempo los arcos quedan divididos en tres partes, lo que permite construir las cuerdas

(c) Consejo Superior de Investigaciones Científicas Licencia Creative Commons 3.0 España (by-nc) correspondientes (arcos y cuerdas resultan simétricos respecto al centro del arco total). Estas cuerdas configurarían una especie de forma poliédrica.

6. Si el ángulo de giro de los arcos (y, por tanto, también de sus cuerdas que son las aristas) es, como en la Fig. 2.4, el adecuado, el arco quedará dividido en tres partes iguales con lo que las cuerdas también serán iguales.

A la figura poliédrica que se obtiene (Fig."2.5) se la ha llamado "viredro" haciendo referencia à que procede de virar las aristas de un poliedro que es su punto de partida. 


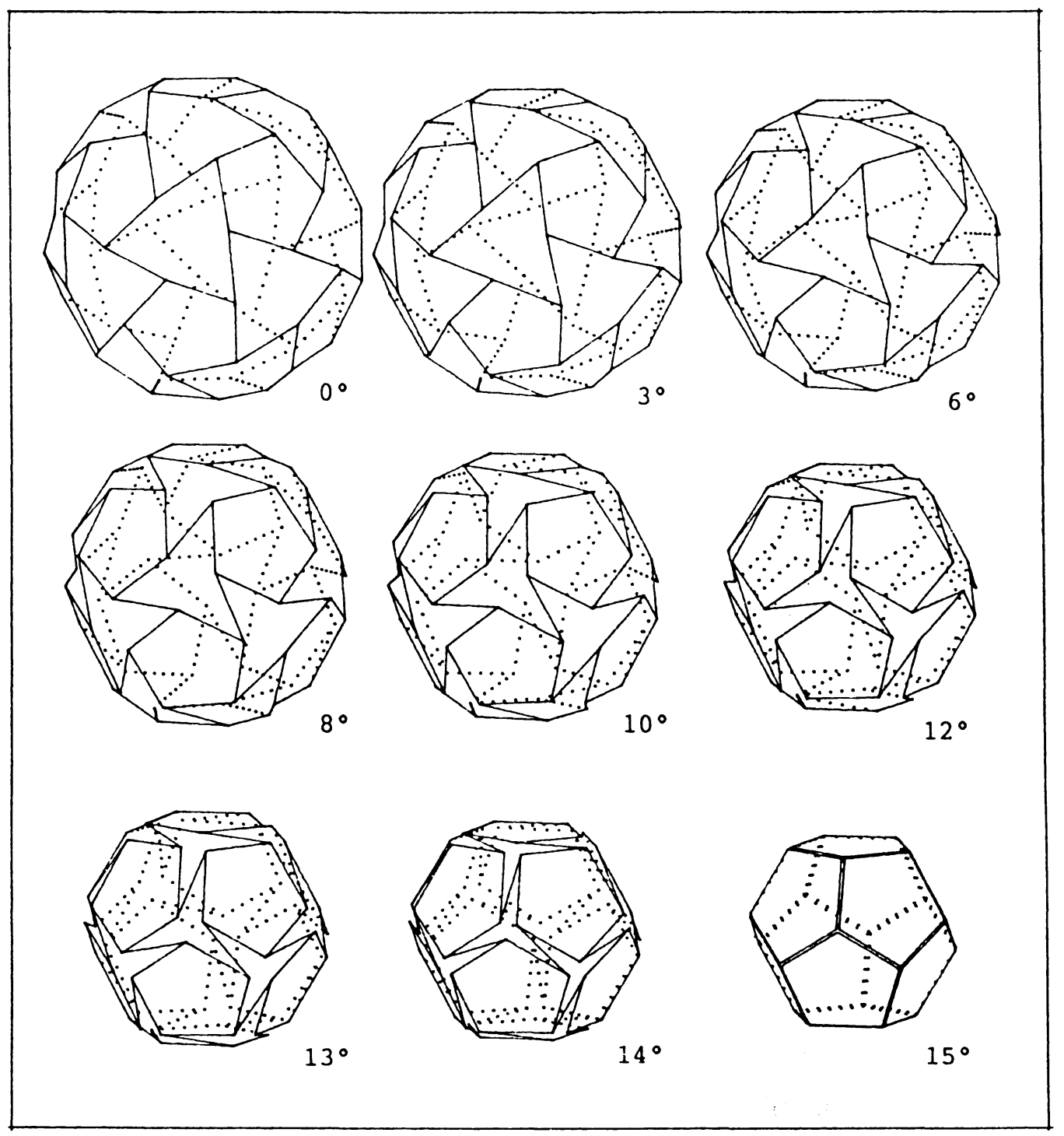

Itg. 3

Este viredro, que tiene todas sus aristas iguales, posee algunas propiedades características. La que nos interesa en particular es la que con articulaciones en sus empalmes puede variar su tamaño de forma coherente, es decir conservando su condición de estar inscrito en una esfera, cuyo radio a su vez también varía.

El estado inicial del proceso lo constituye, pues, el viredro. El estado final es el poliedro regular conjugado del que originó el viredro. En un estado intermedio se pasa por una figura cuyos vértices coinciden con el de un poliedro arquimediano. Fig. 3.

La transformación de un poliedro regular en su conjugado ha sido analizado por varios autores. Entre ellos, F. H. Verheyen (Ref. 2) y R. Williams (Ref. 3). Pero en nuestro caso no es esta transformación de la que se trata, sino la del viredro correspondiente. Y en esta transformación lo que nos interesa no es tanto el paso de un poliedro a otro (es decir, el cambio de forma) sino el cambio de tamaño, puesto que en todo el proceso los poliedros resultantes son inscribibles en una esfera cuyo radio (es decir, cuyo tamaño) va disminuyendo a lo largo de su desarrollo.

En las Figs. 4 a 8 se muestra el proceso para cada uno de los cinco poliedros regulares. En la parte inferior de cada figura aparece la transición entre poliedro origen y su conjugado a partir del viredro correspondiente. Las magnitudes de $\mathrm{R}$ (radio de la esfera circunscrita) expresan lös límites entre los que varía el tamaño real de Tos poliedros. Esta variación secuencial se puede ver para cada uno de los cinco poliedros regulares en la Fig. 9. 
Informes de la Construcción, Vol. 45, n. ${ }^{\circ} 430$, marzo/abril 1994

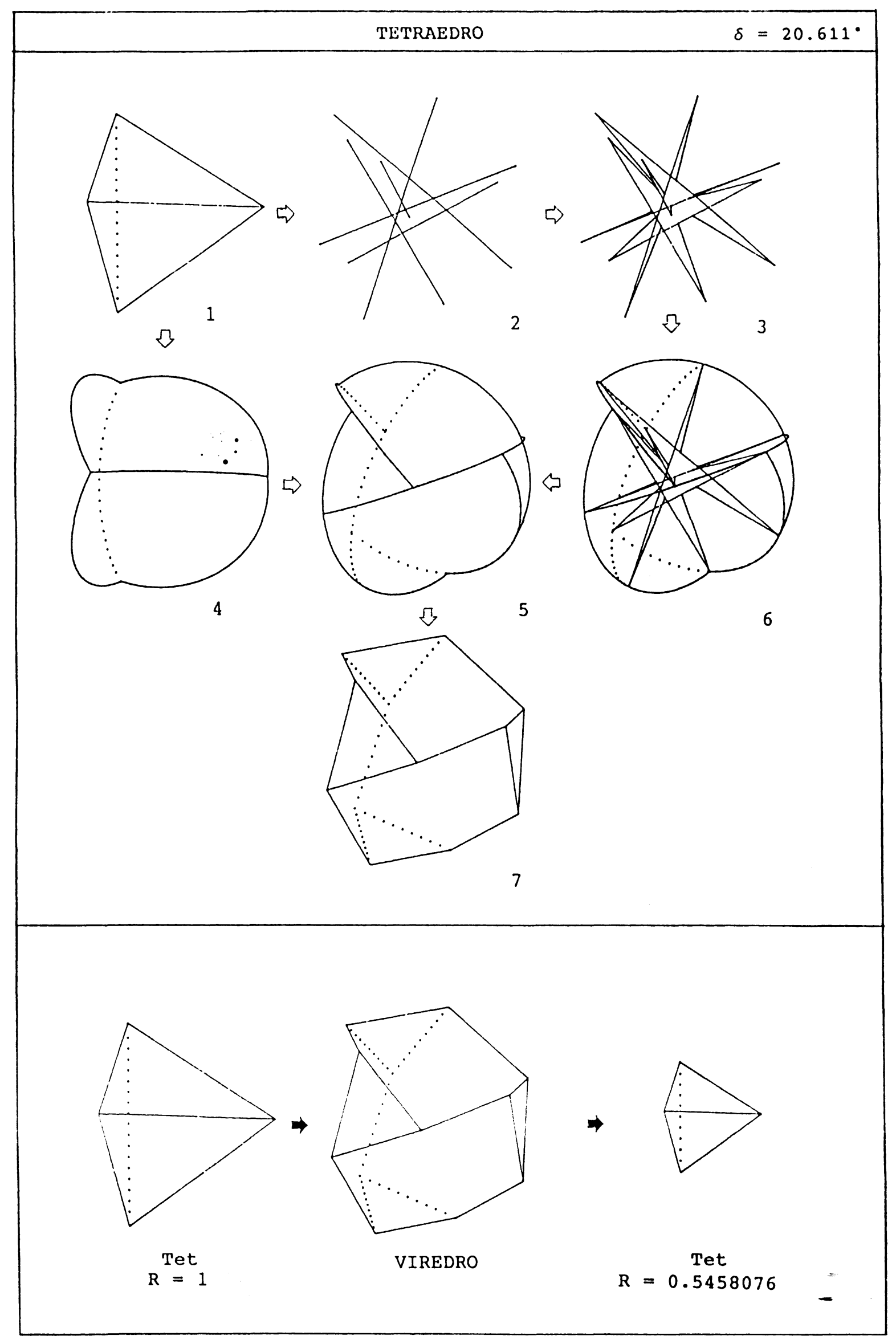

Fig. 4

(c) Consejo Superior de Investigaciones Científicas

Licencia Creative Commons 3.0 España (by-nc)

http://informesdelaconstruccion.revistas.csic.es 


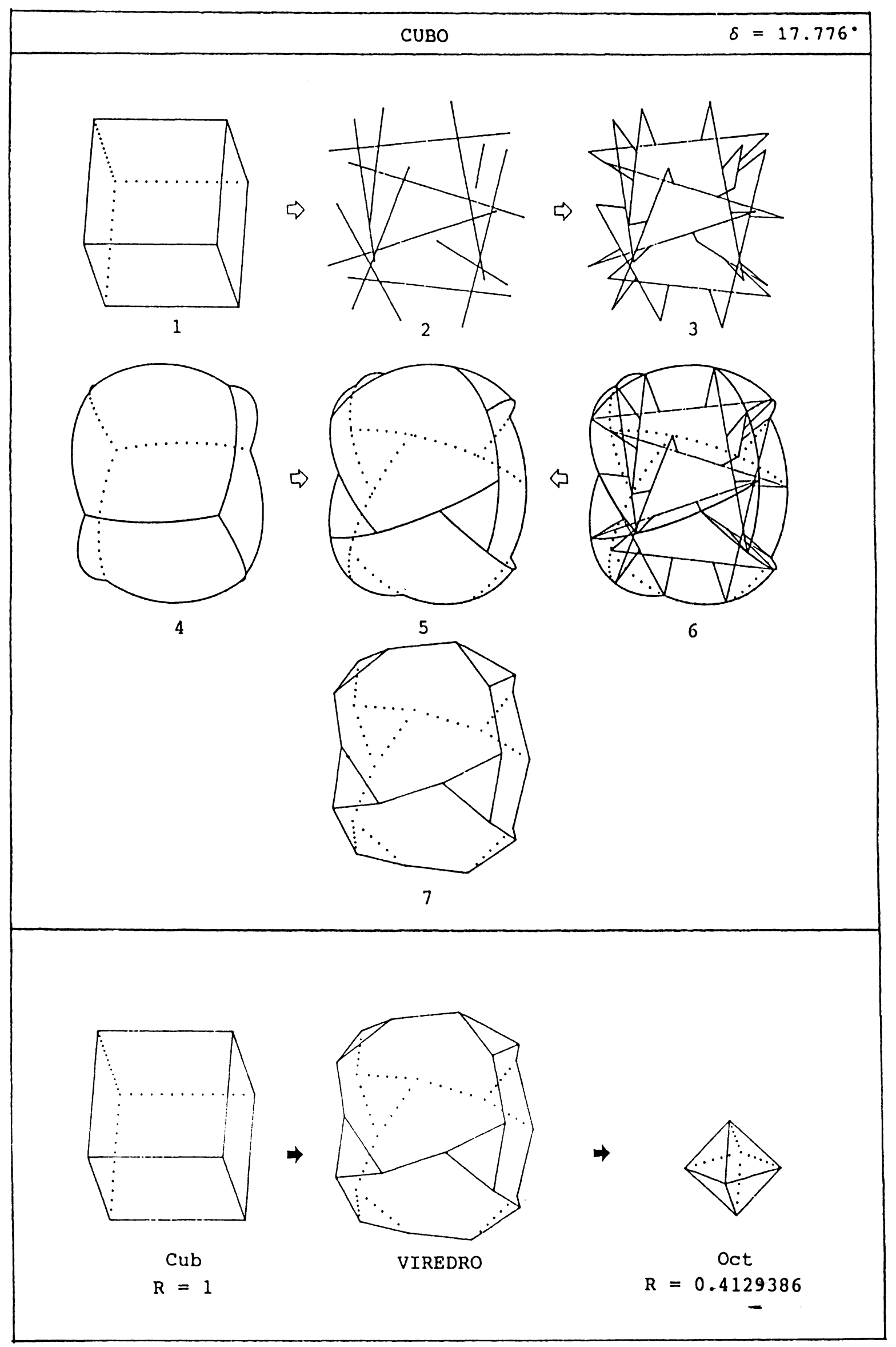

Fig. 5 


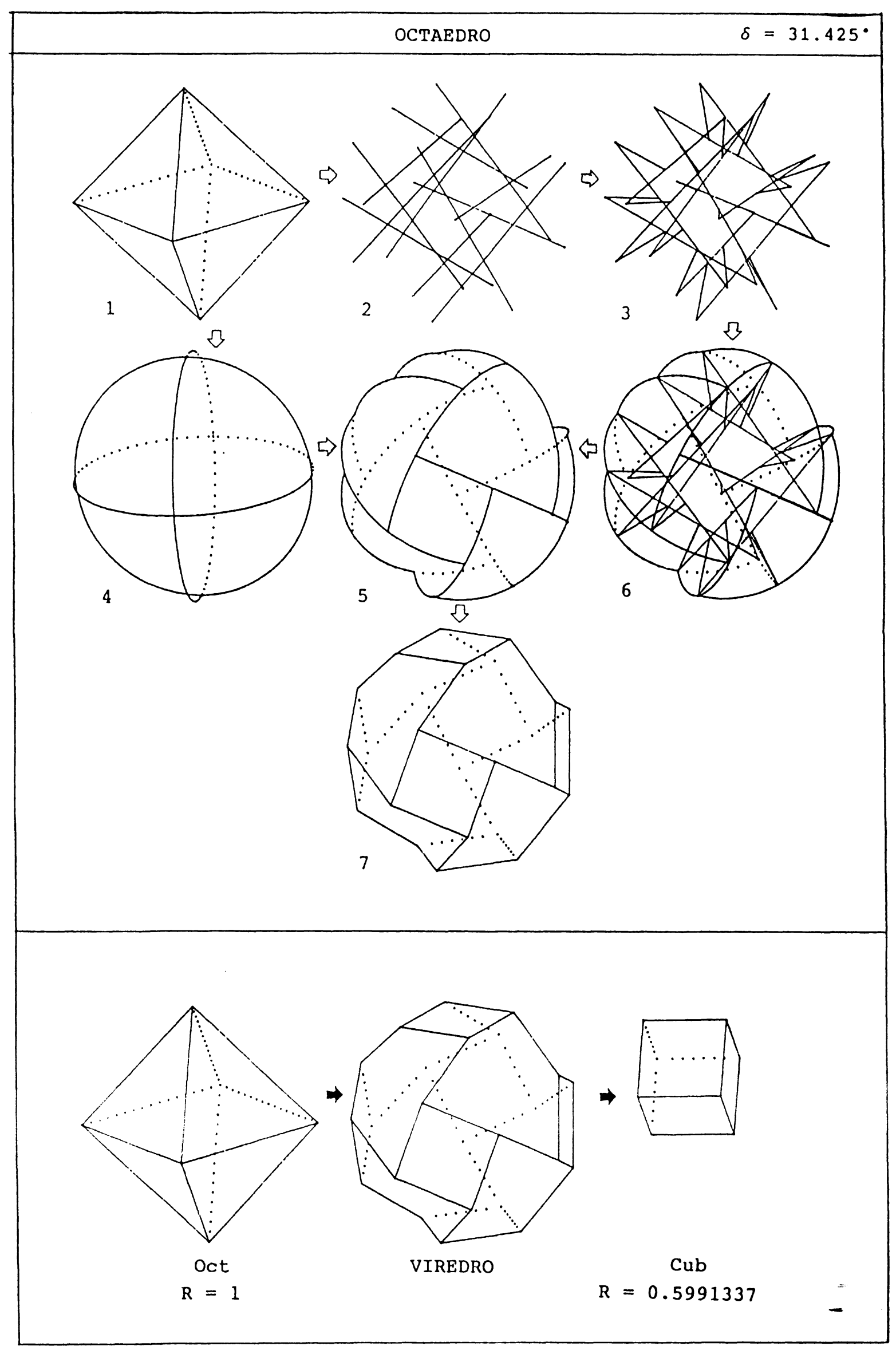




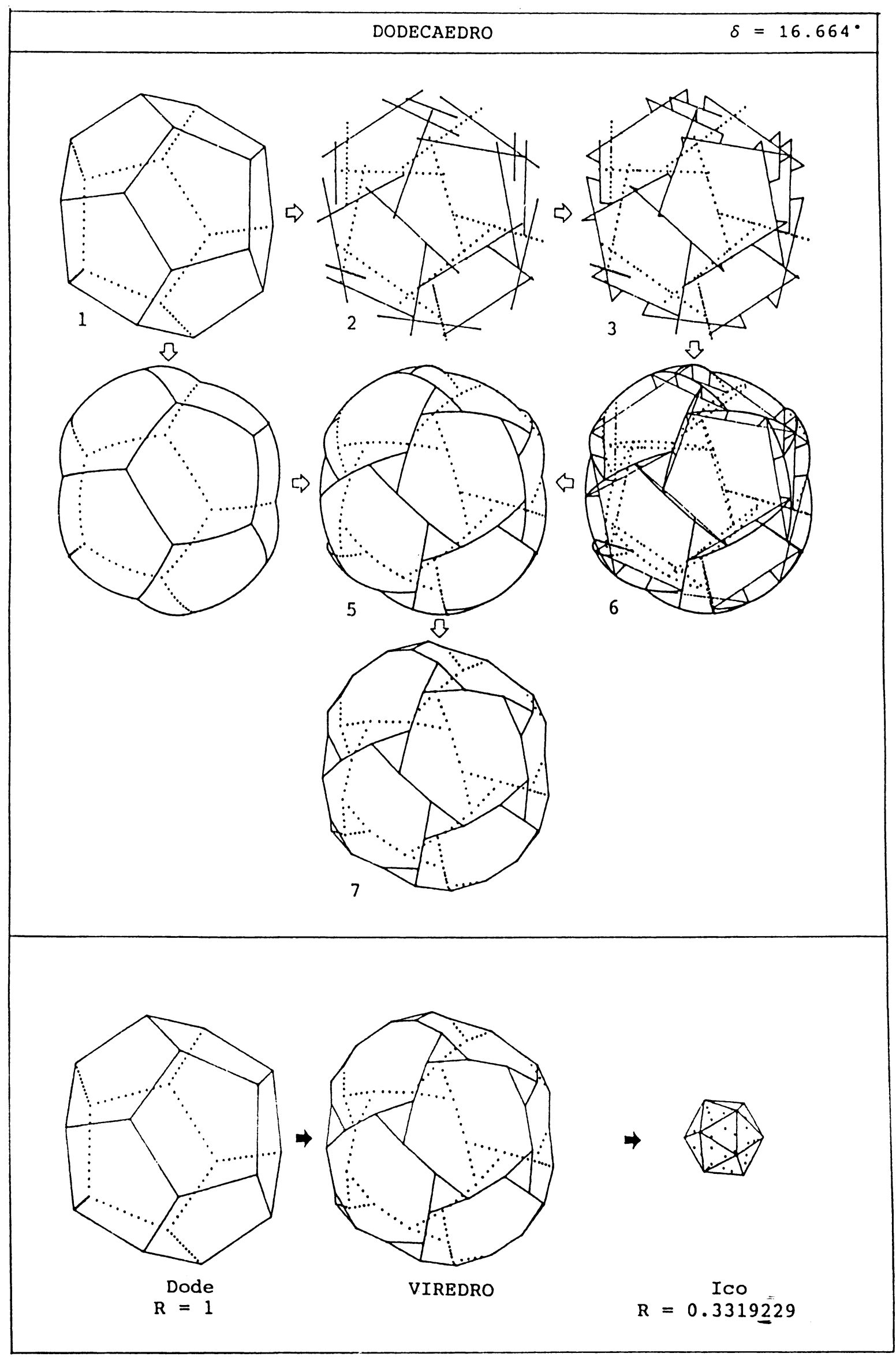




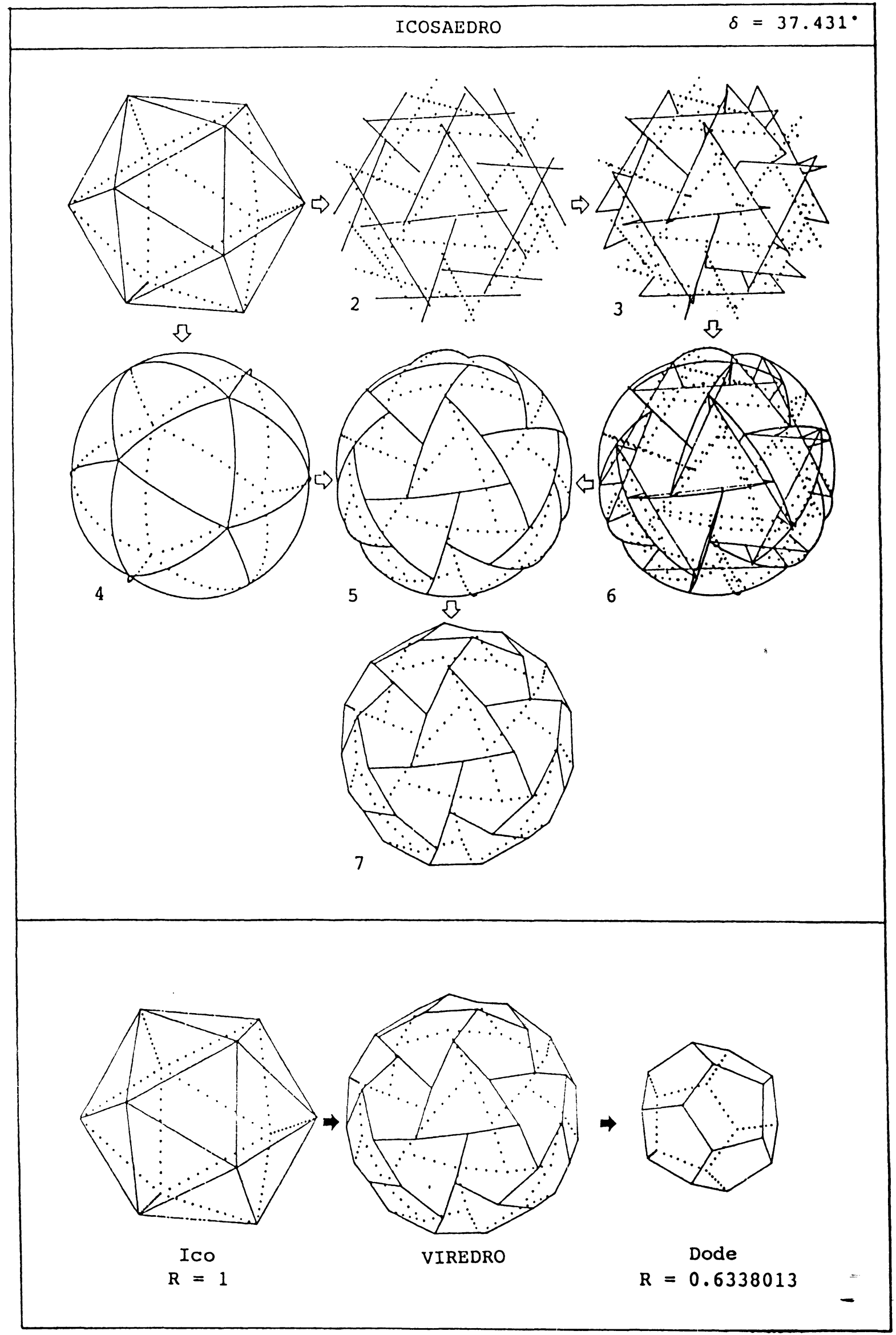




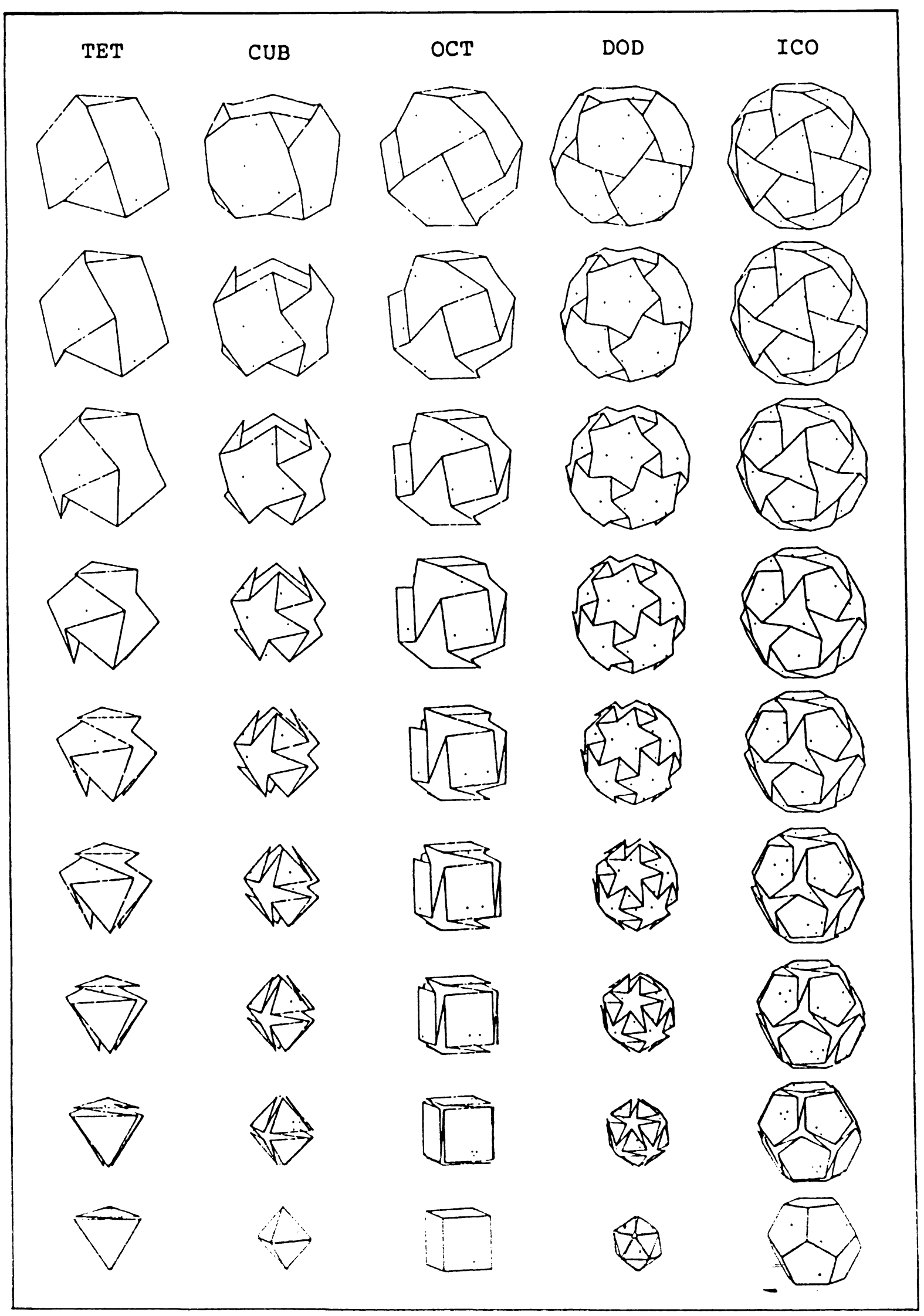

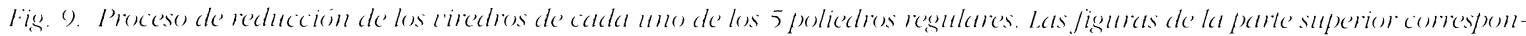
den a los viredros. En cualquiera de las fases de todos los vértices se hallan en la superficie de mina esfera. 


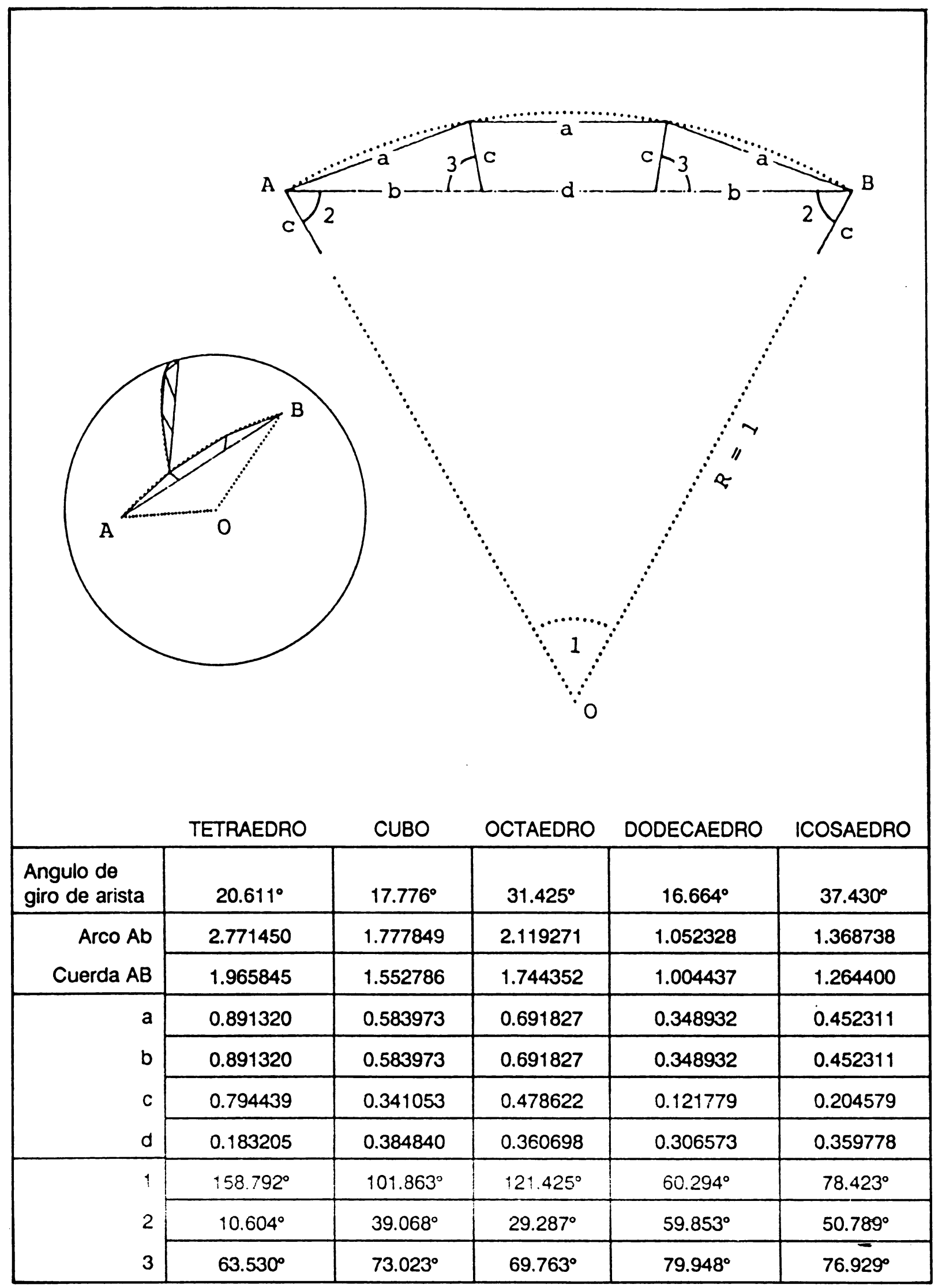

Fig. 10. Cinadro de parametros. 


\section{Conclusiones}

Esta propuesta geométrica tiene dos ventajas respecto a propuestas anteriores. En primer lugar, su extrema sencillez, lo que permite un fácil control de la forma resultante. En segundo lugar, la propuesta queda abierta: es aplicable de inmediato a todas las formas poliédricas regulares, semirregulares y sus conjugados, y también puede generalizarse para los desarrollos geodésicos de cualquiera de los poliedros anteriores, lo que constituirá un trabajo posterior del que la Fig. 11 es una muestra.
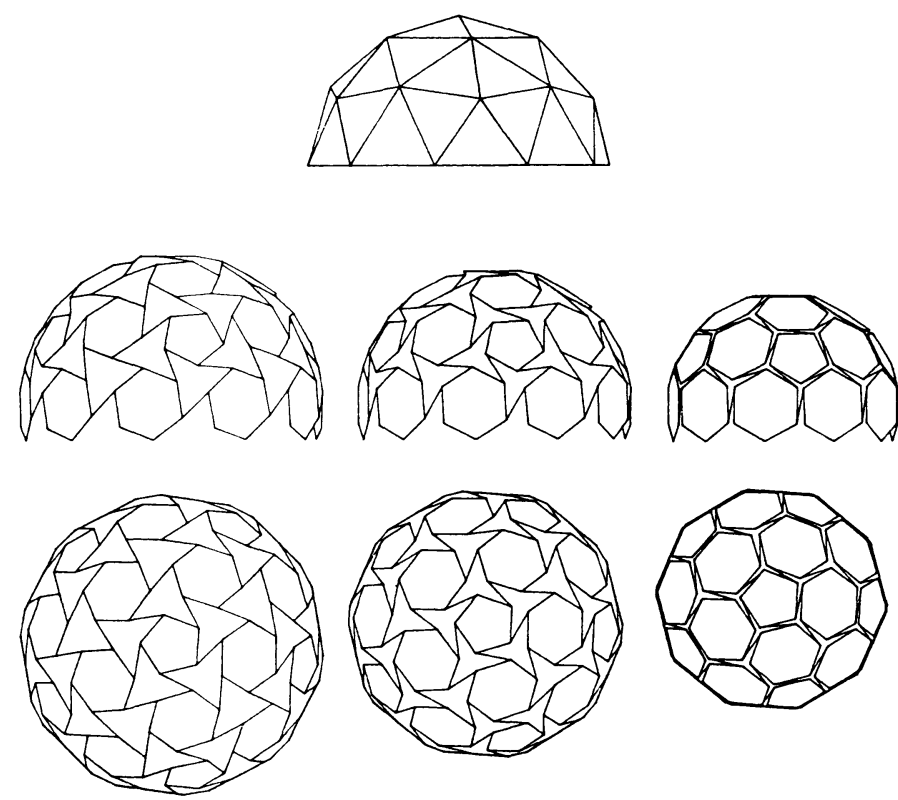

Fig. 11. Lariación del lamaño de una chípula geodésica a partir de su viredro correspondiente. La figura superior corresponde a la geodésica (de base icosaédrica y frecuencia 2). Debajo aparece el viredro en planta y" alzado en tres fases sucesivas.

REFERENCIAS

(1) Mathematical Models, Cundy, H. M. Tarquin Publications, 3. edición, 1981.

(2) Expandable Polyhedral Structures Based on Dipolygonids. Verheyen, H. F. III International Conference on Space Structures. Elsevier Applied Science Publishers, London 1984
(3) The Geometrical Foundation of Natural Structure, Williams, R.: Dover Publications, New York, 1979.

(4) The Formation of Polyhedra by the Rotation of Poligons, Huybers, P.: Space Structure 4, Thomas Telford, London, 1993. 